\title{
Risk factor analysis for central nodal metastasis in papillary thyroid carcinoma
}

\author{
LING-NA MAO $^{1}$, PING WANG $^{2}$, ZHI-YU LI $^{2}$, YONG WANG $^{2}$ and ZHENG-YA SONG ${ }^{1}$ \\ ${ }^{1}$ International Health Care Center; ${ }^{2}$ Department of General Surgery, \\ The Second Affiliated Hospital of Zhejiang University School of Medicine, Hangzhou, Zhejiang 310009, P.R. China
}

Received March 19, 2014; Accepted October 23, 2014

DOI: 10.3892/ol.2014.2667

\begin{abstract}
Lymph node involvement is associated with recurrence in papillary thyroid carcinoma (PTC). The central neck compartment (level VI) lymph nodes are at the greatest risk of metastases from PTC, but the role of central neck dissection (CND) remains controversial, particularly in PTC without clinical cervical lymph node metastasis $\left(\mathrm{cN}_{0}\right)$. The present study aimed to identify risk factors of central cervical nodal metastasis and the safety of CND in patients with $\mathrm{cN}_{0}$ PTC. The current study retrospectively investigated 389 patients who had been followed up for 12.0-25.5 months after surgery, and were divided into positive or negative lymph node involvement groups according to the pathological results subsequent to this surgery. Univariate and multivariate analyses were used to study the risk factor of central node involvement. The mean tumor size was $0.71 \pm 0.35 \mathrm{~cm}$ (range, $0.1-2.0 \mathrm{~cm}$ ). There was no significant difference in the rate of central lymph node involvement based on age ( $<45$ or $\geq 45$ years) or tumor focality (unifocal or multifocal). However, there were significant differences based on gender, extra-thyroid invasion and tumor size $(\mathrm{P}<0.05)$. The incidence of transient hypoparathyroidism and transient vocal cord paralysis following CND was 12.34 and $4.11 \%$, respectively. No patient experienced permanent hypoparathyroidism or vocal cord paralysis. One patient $(1 / 389 ; 0.23 \%)$ experienced disease recurrence during the follow-up. A larger tumor size and the male gender were significantly associated with the central nodal metastasis rate for $\mathrm{cN}_{0}$ PTC with a tumor size of $<2.0 \mathrm{~cm}$. CND for $\mathrm{cN}_{0}$ PTC patients was safe and the tumor-associated recurrence rate following CND plus total thyroidectomy was low. The present study suggests that CND should be conducted for male $\mathrm{cN}_{0}$ PTC patients with a larger tumor size $(\geq 0.5 \mathrm{~cm})$.
\end{abstract}

Correspondence to: Dr Ling-Na Mao, International Health Care Center, The Second Affiliated Hospital of Zhejiang University School of Medicine, 78 Jiefang Road, Hangzhou, Zhejiang 310009, P.R. China

E-mail: borneymao@gmail.com

Key words: papillary thyroid carcinoma, central neck dissection, total thyroidectomy, hypoparathyroidism, vocal cord paralysis

\section{Introduction}

Papillary thyroid carcinoma (PTC) accounts for $>90 \%$ of newly diagnosed thyroid cancers. The incidence of PTC has been increasing in previous decades, without significantly modifying the characteristic low aggressive behavior, in spite of frequent lymph node involvement (1). Overall, 20-50\% of patients with PTC experience cervical lymph node metastasis (2). Usually, metastases from PTC occur in a stepwise fashion from the central to lateral neck compartments. Therefore, the central compartment lymph nodes are at the greatest risk of metastases from PTC. While the effectiveness of therapeutic central neck dissection (CND) is undisputed, there is no consensus on the role of CND in clinically node-negative patients with PTC. Proponents of CND propose that CND offers more accurate staging and may decrease the probability of locoregional recurrence (2-4). Opponents of elective CND highlight the potential for increased morbidity secondary to the risk of recurrent laryngeal nerve injury and hypoparathyroidism $(5,6)$. The overall risk and benefits of the CND procedure must be determined on a patient-by-patient basis. Therefore, the purpose of the present study was to analyze the safety of CND and the risk factors of central nodal metastases in PTC without clinical cervical lymph node metastasis $\left(\mathrm{cN}_{0}\right)$. Written informed consent was obtained from all patients.

\section{Patients and methods}

Patients. The present study was a three-year single institutional retrospective study, approved by the Medical Ethics Committee at The Second Affiliated Hospital of Zhejiang University School of Medicine (Hangzhou, Zhejiang, China). The inclusion criteria of the study were as follows: i) Pathologically confirmed PTC; ii) pre-operative ultrasonography, with or without computed tomography (CT) imaging of the thyroid and cervical lymph node basins; iii) no evidence of nodal disease based on negative physical examination, negative findings on pre-operative neck ultrasonography or CT, or the presence of lyphadenopathy $<5 \mathrm{~mm}$ in diameter $\left(\mathrm{cN}_{0}\right)(7)$; iv) normal vocal cords, confirmed by flexible fiberoptic laryngoscopy, and a normal parathyroid hormone (PTH) level (range, 15-65 pg/ml); v) CND (ipsilateral or bilateral) during planned total thyroidectomy (primary or completion); and 
vi) medical records and histological data available for review. A total of 389 patients who underwent CND for PTC between January 1, 2012 and March 31, 2013 met the inclusion criteria for participation. The exclusion criteria for the study were: i) Previous thyroid or parathyroid surgery; ii) previous neck surgery; iii) previous neck irradiation; iv) concomitant surgery for hyperparathyroidism; and v) surgery for locoregional recurrence.

Surgery. Total thyroidectomy (TT) and CND were conducted by one of three surgeons during the study period, all using a similar technique. The study period was selected to maximize homogeneity of the surgical technique such that $70 \%$ of CNDs were performed by a single surgeon. An ultrasonic scalpel (Harmonic Focus; Johnson \& Johnson, New Brunswick, NJ, USA) was used in cases of hemostasis. Routinely, recurrent laryngeal nerves were identified and exposed until their insertion in the larynx and parathyroid glands were identified and preserved. When devascularization or incidental removal of the parathyroid glands was suspected, a muscular autoimplantation procedure followed. Boundaries of the CND (level VI) were as decided according to the American Thyroid Association classification $(8,9)$. Lymph nodes in this compartment included the pretracheal, paratracheal, prelaryngeal (Delphian) and perithyroidal nodes, including the lymph nodes medial and lateral to the recurrent laryngeal nerves. The superior boundary was defined as the cricoid cartilage, the inferior boundary was the innominate artery and the lateral boundaries were the common carotid arteries. Central neck dissection was performed as either an ipsilateral dissection, on the same side as the primary tumor, or as a bilateral dissection. The pretracheal lymph nodes were included with the ipsilateral CND. In general, bilateral CND was considered for patients with pre-operative or intra-operative evidence of ipsilateral central compartment adenopathy, contralateral central neck adenopathy or bilateral disease in the thyroid.

Following surgery, frequencies and patterns of CND metastases were analyzed with respect to patient characteristics, age and gender, and pathological variables, consisting of tumor size, histological type and primary tumor location (extra-thyroid invasion or not, multifocal or unifocal). For multiple primary lesions, the diameter of the largest dominant tumor was used in the analyses.

Post-operative follow up. All clinical and pathological reports were reviewed. Routine follow-up at one, three and six months post-surgery, and every six months later, included neck ultrasonography and estimation of the serum thyroid stimulate hormone (TSH) level (reference range, 0.55-4.78 mU/1). Indirect laryngoscopy was performed prior to surgery and on the first day after the procedure. Vocal cord paresis that lasted for less than six months post-surgery was regarded as transient, but paresis that persisted for more than six months was regarded as permanent. The total serum calcium level (reference range, 2.08-2.60 mmol/1) was measured $24 \mathrm{~h}$ after surgery and medical treatment was initiated if the concentration was $<2.08 \mathrm{mmol} / \mathrm{l}$. Medication was started prophylactically in order to ensure that no patient developed hypocalcaemia symptoms. When the total serum calcium
Table I. Demographic and pathological data of 389 papilliary thyroid carcinoma patients.

\begin{tabular}{lcc}
\hline Feature & Value & Percentage \\
\hline Patients & & \\
Male & 102 & 26.2 \\
Female & 287 & 73.8 \\
Mean age, years & $42.58 \pm 10.87^{\mathrm{a}}$ &
\end{tabular}

History

Papillary cancer

389

100.0

Tumor

\begin{tabular}{lcc} 
Mean size & $0.71 \pm 0.35^{\mathrm{a}}$ & \\
$\leq 1 \mathrm{~cm}$ & 332 & 85.4 \\
$>1 \mathrm{~cm}$ & 57 & 14.7 \\
Unique & 299 & 76.9 \\
Multifocal & 90 & 23.1 \\
Extrathyroid invasion & 107 & 27.5 \\
Positive LN & 129 & 33.2 \\
\hline
\end{tabular}

${ }^{\mathrm{a}}$ Mean value \pm standard deviation. $\mathrm{LN}$, lymph node.

level was in the range of $1.8-2.08 \mathrm{mmol} / \mathrm{l}$, calcium salts (1.5-3.0 g daily) were administered, and when the level was $<1.8 \mathrm{mmol} / \mathrm{l}$, calcium plus calcitriol (0.25-1.0 $\mu \mathrm{g} /$ day) was administered. The levels of serum calcium, serum phosphate (normal range, 0.81-1.45 $\mathrm{mmol} / \mathrm{l}$ ) and intact PTH (iPTH; chemiluminescence assay; normal range, 15-65 ng/l; detection limit, $6.0 \mathrm{ng} / \mathrm{l}$ ) were determined prior to surgery, on the first day post-surgery and at every follow-up subsequent to the procedure. A serum calcium level of $<2.08 \mathrm{mmol} / 1$ together with a subnormal serum iPTH level $(<15 \mathrm{ng} / \mathrm{l})$ was defined as transient hypoparathyroidism if the level was restored to a normal value within six months after the withdrawal of calcium therapy. Permanent hypoparathyroidism was regarded as persistent hypocalcaemia with a serum iPTH level $<15 \mathrm{ng} / \mathrm{l}$ for more than six months after surgery, which required substitution with calcium, with or without calcitriol.

Statistical analysis. Categorical data were compared using $\chi^{2}$ analysis (univariate analysis) and logistic regression analysis (multivariate analysis) using SPSS Statistics version 18.0 (SPSS, Inc., Chicago, IL, USA). P<0.05 was considered to indicate a statistically significant difference.

\section{Results}

Between January 2012 and March 2013, 389 PTC patients met the inclusion criteria for the present study, 287 females and 102 males (female:male ratio, 2.82:1) with a mean age of $42.58 \pm 10.87$ years. The patients were submitted for TT + CND (Table I). All the patients were successfully followed up until March 2014 (median follow-up, 18.5 months; range, $12.0-25.5$ months). In $14 / 389$ cases (3.60\%), parathyroid 
Table II. Complications in 389 papillary thyroid carcinoma patients.

\begin{tabular}{lrr}
\hline & No. of & \\
Complication & patients & $\%$ \\
\hline Transient hypothyroidism & 48 & 12.34 \\
Permanent hypothyroidim & 0 & 0.00 \\
Parathyroid tissue in the specimen & 14 & 3.60 \\
Transient unilateral vocal cord paralysis & 16 & 4.11 \\
Permanent unilateral vocal cord paralysis & 0 & 0.00 \\
Bilateral vocal cord paralysis & 0 & 0.00 \\
Neck hematoma & 3 & 0.77 \\
\hline
\end{tabular}

Table III. Clinicopathological factors affecting central nodal metastases.

\begin{tabular}{lccc}
\hline Characteristic & $\begin{array}{c}\text { No. of } \\
\text { patients }\end{array}$ & $\begin{array}{c}\text { No. of LN-positive } \\
\text { patients (\%) }\end{array}$ & P-value \\
\hline Age, years & & & \\
$<45$ & 228 & $84(36.8)$ & \\
$\geq 45$ & 161 & $45(28.0)$ & 0.067 \\
Gender & & & \\
Male & 102 & $45(44.1)$ & \\
Female & 287 & $84(29.3)$ & 0.006 \\
Tumor size, cm & & & \\
$\leq 1$ & 332 & $102(30.7)$ & \\
$>1$ & 57 & $27(47.4)$ & 0.014 \\
Extrathyroid invasion & & & \\
Yes & 107 & $44(41.1)$ & \\
No & 282 & $85(30.1)$ & 0.040 \\
Tumor focality & & & 0.289 \\
Unique & 299 & $95(31.8)$ & \\
Multifocal & 90 & $34(37.8)$ & \\
\hline
\end{tabular}

LN, lymph node.

tissue was identified in the final histopathological analysis. The incidence of surgical complications is reported in Table II. In three patients $(0.77 \%)$, a neck hematoma that required surgical re-exploration was observed. During the follow-up, only one patient $(1 / 389 ; 0.26 \%)$, a 22 -year-old female, experienced disease recurrence in the residual thyroid gland and received an additional surgery 18 months after the initial procedure. None of the patients experienced further metastasis or tumor-associated mortality.

The mean tumor size was $0.71 \pm 0.35 \mathrm{~cm}$ (range, $0.1-2 \mathrm{~cm}$ ) and microcarcinoma (tumor size, $\leq 1 \mathrm{~cm}$ ) was diagnosed in 332 patients $(85.35 \%)$. The fact that all the tumors included were $\leq 2.0 \mathrm{~cm}$ was incidental. The histotype was papillary carcinoma in all patients. A total of 90 patients $(21.14 \%)$
Table IV. Clinicopathological factors affecting central nodal metastases in microcarcinoma patients $(n=332)$.

\begin{tabular}{lccc}
\hline Characteristic & $\begin{array}{c}\text { No. of } \\
\text { patients }\end{array}$ & $\begin{array}{c}\text { No. of LN-positive } \\
\text { patients, } \%\end{array}$ & $\mathrm{P}_{1}$-value \\
\hline $\begin{array}{l}\text { Age, years } \\
<45\end{array}$ & 201 & $67(33.3)$ & \\
$\geq 45$ & 131 & $35(26.7)$ & 0.202 \\
Gender & & & \\
Male & 85 & $36(42.4)$ & \\
Female & 247 & $66(26.7)$ & 0.007 \\
Tumor size, cm & & & \\
$\leq 0.5$ & 144 & $31(21.5)$ & \\
$>0.5$ & 188 & $71(37.8)$ & 0.001 \\
Extrathyroid invasion & & & 0.039 \\
Yes & 80 & $32(40.0)$ & \\
No & 252 & $70(27.8)$ & \\
Tumor focality & & & \\
Unique & & & \\
Multifocal & 255 & $74(29.0)$ & \\
\hline LN, lymph node. & 77 & $28(36.4)$ & \\
\hline
\end{tabular}

Table V. Multivariate logistic regression analysis of central lymph node involvement.

\begin{tabular}{lcrrrrrr}
\hline & \multicolumn{6}{c}{ Variables in the equation } \\
\cline { 2 - 7 } Risk factors & B & S.E. & Wald & df & Sig. & Exp(B) \\
\hline Gender & 0.650 & 0.246 & 7.016 & 1 & 0.008 & 1.916 \\
Tumor size & 1.319 & 0.337 & 15.288 & 1 & 0.000 & 3.738 \\
Extrathyroid & 0.182 & 0.255 & 0.510 & 1 & 0.475 & 1.200 \\
invasion & & & & & & \\
Constant & -1.907 & 0.279 & 46.848 & 1 & 0.000 & 0.149
\end{tabular}

B, coefficient of regression; S.E., standard error of B; Wald, statistical value $\left(\chi^{2}\right)$ used for hypothesis testing of $B$; df, degrees of freedom; Sig., P-value; $\operatorname{Exp}(\mathrm{B})$, adjusted odds ratio.

possessed multifocal tumors, 107 patients (27.51\%) were found with tumor extrathyroid invasion and lymph node involvement was identified in 129 patients (33.16\%) (Table I).

Clinicopathological factors affecting central nodal metastases in patients were assessed by univariate analysis and are summarized in Table III for patients with conventional PTC and in Table IV for patients with a tumor size of $\leq 1.0 \mathrm{~cm}$. There was no significant difference between patients with central nodal metastases with respect to age $[\mathrm{P}=0.067$; $\mathrm{P}$-value for microcarcinoma $\left.\left(\mathrm{P}_{1}\right)=0.202\right]$ or tumor focality $(\mathrm{P}=0.289$; $\left.P_{1}=0.221\right)$. However, there were significant differences between patients with central nodal metastases with respect to gender 
Table VI. Multivariate logistic regression analysis of central lymph node involvement in microcarcinoma.

\begin{tabular}{|c|c|c|c|c|c|c|}
\hline \multirow[b]{2}{*}{ Risk factors } & \multicolumn{6}{|c|}{ Variables in the equation } \\
\hline & B & S.E. & Wald & $\mathrm{df}$ & Sig1. & $\operatorname{Exp}(B)$ \\
\hline Gender & 0.760 & 0.271 & 7.832 & 1 & 0.005 & 2.137 \\
\hline $\begin{array}{l}\text { Tumor } \\
\text { size, cm }\end{array}$ & 1.958 & 0.572 & 11.708 & 1 & 0.001 & 7.086 \\
\hline $\begin{array}{l}\text { Extrathyroid } \\
\text { invasion }\end{array}$ & 0.235 & 0.292 & 0.648 & 1 & 0.421 & 1.265 \\
\hline Constant & -2.312 & 0.388 & 35.532 & 1 & 0.000 & 0.099 \\
\hline
\end{tabular}

B, coefficient of regression; S.E., standard error of B; Wald, statistical value $\left(\chi^{2}\right)$ used for hypothesis testing of $\mathrm{B}$; df, degrees of freedom; Sig1., P-value; $\operatorname{Exp}(\mathrm{B})$, adjusted odds ratio.

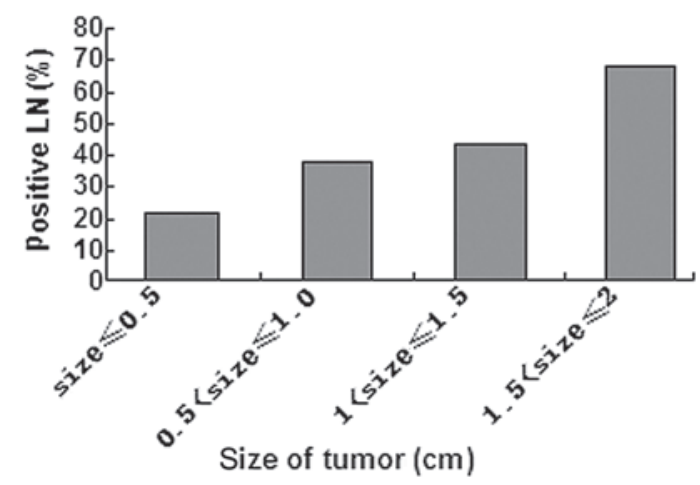

Figure 1. Different rate of central node metastasis in varying tumor size groups. The rates were $21.5,37.8,43.8,67.8 \%$, from left to right accordingly. LN, lymph node.

$\left(\mathrm{P}=0.006 ; \mathrm{P}_{1}=0.007\right)$, tumor size $\left(\mathrm{P}=0.014 ; \mathrm{P}_{1}=0.001\right)$ and extrathyroid invasion $\left(\mathrm{P}=0.04 ; \mathrm{P}_{1}=0.039\right)$.

In the multivariable analysis, the male gender and tumor size were found to be significantly associated with central lymph node metastasis for all the patients in the study (Tables V and VI).

From the statistics, it was indicated that compared with females, males were more vulnerable to developing positive lymph node involvement in the present study. The incidence of central lymph node metastasis appeared to be higher with a larger tumor size. The correlation coefficient between tumor size and central lymph node metastasis was 0.228 , which was calculated using a two-tailed Pearson product-moment correlation $(\mathrm{P}<0.01)$.

\section{Discussion}

Differentiated PTC exhibits a high propensity to spread to regional lymph nodes. As aforementioned, the reported incidence of clinically positive lymph nodes ranges between 20 and $50 \%$ (2), and was $33.2 \%$ in the present study. A higher proportion $(80-90 \%)$ of patients exhibit subclinical lymph node metastases (micrometastases) at the time of surgical intervention (10-12). Despite the high incidence, lymph node metastases are not considered prognostic for poor survival in patients with well-differentiated PTC (13). Therefore, treatment of the cervical lymph nodes in well-differentiated PTC remains controversial. The primary argument for performing CND in the treatment of well-differentiated PTC is to more accurately stage the tumor. Accurate staging allows for improved risk stratification and the more rational application of levothyroxine suppression and adjuvant therapy, such as iodine ${ }^{131}$ I ablation (2-4). The presence or absence of pathological lymph nodes in neck dissection specimens has been reported to correlate with the incidence of disease recurrence. Elective CND may aid in the prevention of local recurrences in the central compartment where re-operation can be challenging (2-4). Therefore, there are proponents and opponents of elective CND. Certain PTC lesions ( $25 \%)$, particularly in the older patient population ( $\geq 45$ years old), concentrate radioactive iodine poorly (14-16). In these cases, radioactive iodine treatment may not adequately treat residual nodal micrometastasis. Thus, it is likely that elective CND is most beneficial at the time of initial surgery for selected high-risk patients.

Determining the patients that are high risk prior to surgery remains difficult. The present study aimed to identify factors associated with central neck compartment nodal metastases as an initial step toward defining the patients that are most likely to benefit from elective CND. Malignant lymph nodes were found to occur with high frequency in male patients with a larger tumor size or extrathyroid invasion. Overall, from the statistical analysis, tumor size was found to be an extremely significant risk factor of central lymph node involvement in PTC. There was a significant difference $(\mathrm{P}<0.05)$ in the incidence of central lymph node metastasis between the four groups (Fig. 1) and the larger the tumor size, the higher the incidence. Only when the tumor size was $\leq 0.5 \mathrm{~cm}$, was the incidence of lymph node involvement $<30 \%$.

Post-operative complications in the present study were comparative to those of other studies and to TT alone without CND (17-20). None of the patients underwent permanent hypoparathyroidism or permanent vocal cord paralysis. During the follow-up period, only one patient experienced recurrence. It appeared that CND did not increase the chance of the loco-regional recurrence. The safety of the surgery may partly be due to the high volume of surgeries and the experience of the surgeons at the Department of General Surgery at The Second Affiliated Hospital of Zhejiang University School of Medicine, with $>1,000$ cases treated per year. Therefore, the present conclusion is that it is advisable for male PTC patients $\left(\mathrm{cN}_{0}\right)$ with a tumor size of $\geq 0.5 \mathrm{~cm}$ to receive prophylactic CND, particularly when performed by a surgeon that treats a high volume of PTC cases.

There are certain limitations to the present study. First, all the procedures were conducted by three surgeons. Although they were all surgeons who treat high volumes of PTC cases and used a similar technique, there are differences between them with regard to the extent of CND and the safety of the surgery. Second, the follow-up period was not long enough. Complications, particularly the recurrence rate or mortality rate due to PTC may change over time. The third limitation may also have been an advantage for the present study, as the tumor size was refined to $2.0 \mathrm{~cm}$. If possible, an increased number of patients with larger tumor sizes should be included in the future. Further observation and study are required to 
better define the risk factors of central lymph node metastasis and the safety of CND in $\mathrm{cN}_{0}$ PTC.

\section{Acknowledgements}

The authors would like to thank Dr. Ping Wang's surgery team for their hard work during the procedures and follow-up period. The authors would also like to thank the patients who were involved in the study.

\section{References}

1. Pacini F: Changing natural history of differentiated thyroid cancer. Endocrine 42: 229-230, 2012.

2. Cooper DS, Doherty GM, Haugen BR, et al; American Thyroid Association Guidelines Taskforce: Management guidelines for patients with thyroid nodules and differentiated thyroid cancer. Thyroid 16: 109-142, 2006.

3. Shindo M, Wu JC, Park EE and Tanzella F: The importance of central compartment elective lymph node excision in the staging and treatment of papillary thyroid cancer. Arch Otolaryngol Head Neck Surg 132: 650-654, 2006.

4. White ML, Gauger PG and Doherty GM: Central lymph node dissection in differentiated thyroid cancer. World J Surg 31: 895-904, 2007.

5. Shaha AR: Management of the neck in thyroid cancer. Otolaryngol Clin North Am 31: 823-831, 1998.

6. Mazzaferri EL and Jhiang SM: Long-term impact of initial surgical and medical therapy on papillary and follicular thyroid cancer. Am J Med 97: 418-428, 1994.

7. Kowalski LP, Bagietto R, Lara JR, et al: Prognostic significance of the distribution of neck node metastasis from oral carcinoma. Head Neck 22: 207-214, 2000.

8. American Thyroid Association (ATA) Guidelines Taskforce on Thyroid Nodules and Differentiated Thyroid Cancer; Cooper DS Doherty GM, Haugen BR, et al: Revised American Thyroid Association management guidelines for patients with thyroid nodules and differentiated thyroid cancer. Thyroid 19: 1167-1214, 2009.
9. American Thyroid Association Surgery Working Group; American Association of Endocrine Surgeons; American Academy of Otolaryngology-Head and Neck Surgery; American Head and Neck Society; Carty SE, Cooper DS, Doherty GM, et al: Consensus statement on the terminology and classification of central neck dissection for thyroid cancer. Thyroid 19: 1153-1158, 2009.

10. Chow SM, Law SC, Chan JK, et al: Papillary microcarcinoma of the thyroid - prognostic significance of lymph node metastasis and multifocality. Cancer 98: 31-40, 2003.

11. Hay ID, Grant CS, van Heerden JA, et al: Papillary thyroid microcarcinoma: a study of 535 cases observed in a 50-year period. Surgery 112: 1139-1146, 1992.

12. Qubain SW, Nakano S,Baba M, Takao S and Aikou T: Distribution of lymph node micrometastasis in pNO well-differentiated thyroid carcinoma. Surgery 131: 249-256, 2002.

13. Shaha AR: Implications of prognostic factors and risk groups in the management of differentiated thyroid cancer. Laryngoscope 114: 393-402, 2004.

14. Schlumberger M, Challeton C, De Vathaire F, et al: Radioactive iodine treatment and external radiotherapy for lung and bone metastasis from thyroid carcinoma. J Nucl Med 37: 598-605, 1996.

15. Sawka AM, Brierley JD, Tsang RW, et al: An updated systemic review and commentary examining the effectiveness of radioactive iodine remnant ablation in well-differentiated thyroid cancer. Endocrinol Metab Clin North Am 37: 457-480, 2008.

16. Ganti AK and Cohen EE: Iodine-refractory thyroid carcinoma. Rev Recent Clin Trials 1: 133-141, 2006.

17. Hughes DT, White ML, Miller BS, et al: Influence of prophylactic central node dissection on postoperative thyroglobulin levels and radioiodine treatment in papillary thyroid cancer. Surgery 148: 1100-1106, 2010

18. Poppadich A, Levin O, Lee JC, et al: A multicenter cohort study of total thyroidectomy and routine central lymph node dissection for $\mathrm{cN}_{0}$ papillary thyroid cancer. Surgery 150: 1048-1057, 2011.

19. Giordano D, Valcavi R, Thompson GB, et al: Complications of central neck dissection in patients with papillary thyroid carcinoma: results of a study on 1087 patients and review of the literature. Thyroid 22: 911-917, 2012.

20. Barczyński M, Konturek A, Stopa M and Nowak W: Prophylactic central neck dissection for papillary thyroid cancer. Br J Surg 100: 410-418, 2013. 